\begin{tabular}{|c|c|}
\hline Title & Copper(I)-Catalyzed Boryl Substitution of A llyl A minals: Selective Synthesis of Linear gamma A minoally lboronates \\
\hline Author(s) & Takenouchi, Y uta; Ito, Hajime \\
\hline Citation & $\begin{array}{l}\text { Synthesis stuttgart, 49(21), } 4738-4744 \\
\text { https://doi.org/10.1055/s } 0036-1588802\end{array}$ \\
\hline Issue Date & $2017-11$ \\
\hline Doc URL & http:/hdl .handle.net/2115/71764 \\
\hline Rights & (c) 2017 Georg Thieme V erlag \\
\hline Type & article (author version) \\
\hline File Information & A minal_14.pdf \\
\hline
\end{tabular}

Instructions for use 


\section{Copper(I)-Catalyzed Boryl Substitution of Allyl Aminals: Selective Synthesis of Linear $\gamma$-Aminoallylboronates}

\author{
Y. Takenouchi \\ H. Ito* \\ Division of Applied Chemistry and Frontier Chemistry Center, \\ Faculty of Engineering, Hokkaido University, Sapporo, \\ Hokkaido, 060-8628, Japan. \\ E-mail: hajito@eng.hokudai.ac.jp
}

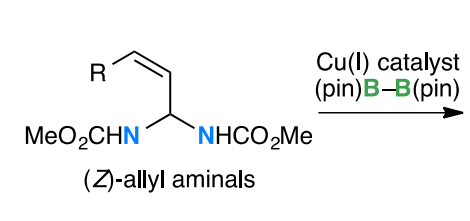

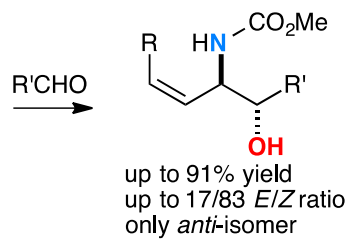

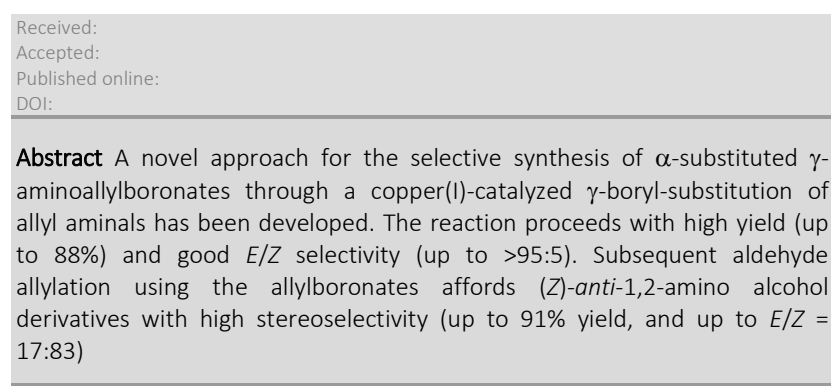

Key words: allylboronate, copper, catalysis, 1,2-amino alcohol, allylboration

1,2-Amino alcohols are common structural motifs in natural products and pharmaceuticals. ${ }^{1}$ Establishing an efficient synthesis of this motif is an important challenge, and various synthetic approaches have been developed to obtain vicinal amino alcohols. ${ }^{2}$ Among them, approaches involving formation of an amino alcohol moiety along with a new C-C bond are particularly attractive, such as the convergent synthesis of 1,2amino alcohol derivatives that have a complex carbon skeleton. ${ }^{3}$ Although the allylation of carbonyl compounds with $\gamma$-aminosubstituted allylboron compounds has great potential for accessing stereo-defined vicinal amino alcohol derivatives due to their high levels of chemo- and diastereo-selectivities, synthetic routes to $\gamma$-aminoallylboron compounds are limited: lithiation of allyl amines and subsequent reaction with boron electrophiles (Scheme 1a). ${ }^{4}$ Recently, a Pd-catalyzed reaction for the synthesis of these boronates has been reported by Hall and co-workers, but this method is only suitable for the synthesis of six-membered heterocyclic products, and is not applicable to linear $\gamma$-aminoallylboronates (Scheme 1b). ${ }^{5}$ Development of a catalytic method for the preparation of linear $\gamma$ aminoallylboronates is therefore still required. a) Classical Preparation of Linear $\gamma$-Aminoallylboronates

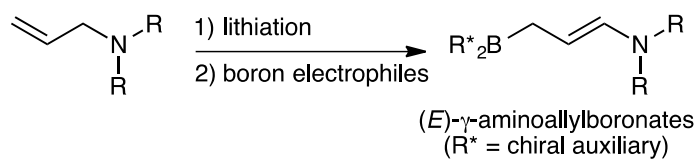

b) Catalytic Preparation of Cyclic $\gamma$-Aminoallylboronate (Hall)

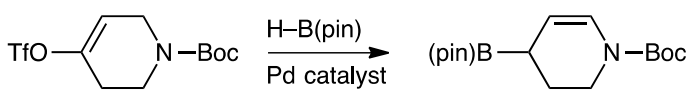

(Z)- $\gamma$-aminoallylboronate

Scheme 1 Preparation Methods for $\gamma$-Aminoallylboronates

Copper(I)-catalyzed boryl substitution is an efficient way to obtain allylboron compounds due to its high regioselectivity and functional group tolerance. Recently, our group succeeded in the development of an enantioselective boryl substitution of allyl acetals 1a to give enantio-enriched $\gamma$-alkoxyallylboronates. ${ }^{6}$ This transformation provided a highly stereoselective allylation of aldehydes with $\gamma$-alkoxyallylborontes to afford chiral 1,2-diol derivatives (Scheme 2a). Extending the scope of the copper(I)catalyzed boryl substitution/aldehyde allylation procedure, we focused on readily available allyl aminals as substrates. When the one of the amino groups in the allyl aminal acts as the leaving group in the copper(I)-catalyzed allylic boryl substitution, $\gamma$-aminoallylboronates will be formed. Although copper catalysis has been widely used for nucleophilic boryl substitutions of allyl and propargyl carbonates, ${ }^{7}$ ethers, ${ }^{8}$ sulfonates, ${ }^{9}$ and phosphates, ${ }^{10}$ to the best of our knowledge, the reaction using a nitrogen-containing functional group as a leaving group has been limited to strained compounds such as propargyl aziridines $\mathbf{1} \mathbf{b}^{11}$ or diazabicycles $\mathbf{1} \mathbf{c}^{12}$ (Schemes $2 \mathrm{~b}$ and $2 c$ ). It was not clear whether the amino group could act as a leaving group, but we anticipated that it would be possible by thorough optimization of the reaction conditions. 


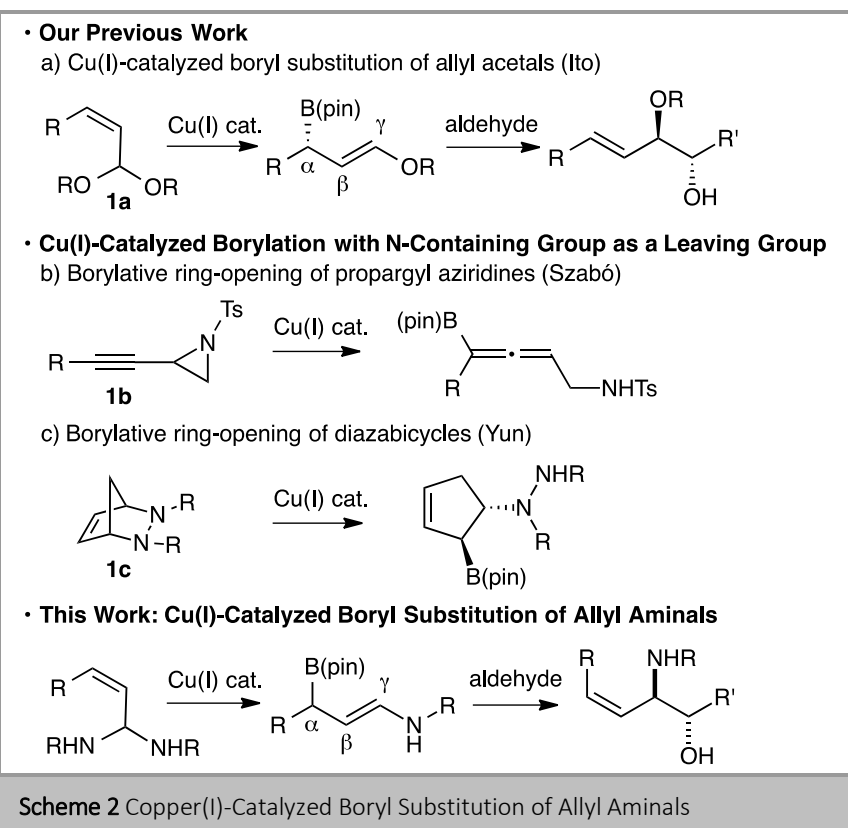

Herein, we report a novel synthetic approach for the synthesis of linear $\gamma$-aminoallylboronates via copper(I)catalyzed boryl substitution of allyl aminals. Borylation of $\mathrm{N}$ methylcarbamate-protected allyl aminals afforded the corresponding boronates in high yield and $E / Z$ selectivity (54$88 \%$ yield, and up to $>95: 5 E / Z$ ratio). The allylation of aldehydes with the allylboronates afforded the desired 1,2amino alcohol derivatives in good yield and $E / Z$ selectivity (76\%-91\% yield, and up to $17: 83 E / Z$ ratio).

Allyl aminal-(Z)-2a, with $N$-methyl carbamate protecting groups, was prepared prior to reaction optimization. The allyl aminals were easily prepared using the procedure reported by Maruoka. ${ }^{13}$ The reaction of aminal (Z)-2a with $\mathrm{B}_{2}(\mathrm{pin})_{2}$ in the presence of $\mathrm{CuCl} / \mathrm{dppbz}$ as catalyst (5 $\mathrm{mol} \%$ ) and $\mathrm{KOMe}$ as a base (1 equiv) in THF afforded the desired allylboronate $(E)$-3a in high yield and $E / Z$ selectivity (Table 1, entry 1,89\%). The borylation of $(Z)-2 a$ in the absence of a ligand provided the corresponding product in poor yield (entry 2). The reaction with Xantphos, which is an effective diphosphine ligand in many copper(I)-catalyzed borylation reactions, ${ }^{7-10}$ was also found to give the product in poor yield (entry 3 ). The use of alternative bidentate phosphine ligands such as dppe, DPEphos or dmpe resulted in poor to moderate yields (entries 4-6). The use of phenanthroline, NHC, or monodentate $\mathrm{PPh}_{3}$ ligands afforded inferior results compared with that of the dppbz ligand (entries 7-9). Addition of a proton source such as $\mathrm{MeOH}$ or $t$ - $\mathrm{BuOH}$ was necessary for protonation of the in situ-generated amino-copper species in the borylative ring-opening (Schemes $2 \mathrm{~b}$ and $2 \mathrm{c}$ ). ${ }^{11,12}$ Thus, we investigated effect of adding an alcohol to this reaction, but the addition of $\mathrm{MeOH}$ afforded only a trace mount of product (entry 10). We then studied the effect of the base on the reactivity. The use of $\mathrm{K}(\mathrm{O}-\mathrm{t}-\mathrm{Bu})$ or $\mathrm{NaOMe}$ resulted in moderate yields (entries 11 and 12). Finally, the impact of the protecting group on the substrate was evaluated by the borylation reactions of $N$-Boc-protected aminal $(Z)-2 \mathbf{b}$ or $N$-Cbz-protected aminal (Z)-2c. These substrates afforded the corresponding product in poor yield (entries 13 and 14).
Table 1 Optimization of the Reaction Conditions for the Copper(I)-Catalyzed Boryl Substitution of Allyl Aminals (Z)-2 ${ }^{a}$

\begin{tabular}{|l|l|l|l|c|}
\hline & \\
\hline
\end{tabular}

${ }^{a}$ Reagents and conditions: $\mathrm{CuCl}(0.0125 \mathrm{mmol})$, ligand $(0.0125 \mathrm{mmol}),(Z)-2(0.25$ $\mathrm{mmol})$, bis(pinacolato)diboron $(0.375 \mathrm{mmol})$ and base $(0.25 \mathrm{mmol})$ in solvent $(0.75$ $\mathrm{mL}$ ) at $30^{\circ} \mathrm{C} .{ }^{b} \mathrm{GC}$ yield. ${ }^{c}$ Isolated yield. ${ }^{d} 10 \mathrm{~mol} \%$ of $\mathrm{PPh}_{3}$ was used. ${ }^{e} 0.3$ equiv of $\mathrm{KOMe}$ and 2.0 equiv of $\mathrm{MeOH}$ were used. ${ }^{f} \mathrm{NMR}$ yield. ${ }^{g} 54 \mathrm{~h}$.

With the optimized conditions in hand (Table 1, entry 1), we then started to explore the scope of the aminals. Various $\gamma$ aminoallylboronates were obtained using the optimized conditions (Scheme 3). The borylation of aminal (Z)-2d, with a terminal olefin, proceeded in high yield with poor $E / Z$ selectivity ( $88 \%$ yield, $E / Z=3: 1$ ). The poor $E / Z$ selectivity was caused by the flexible conformation of the substrate. ${ }^{6}$ The methyl group played an important role in enhancing the $E / Z$ selectivity illustrated by boryl substitution of (Z)-2e yielding the corresponding product in $70 \%$ yield with perfect $E / Z$ selectivity. Boronate $(E)$-3f, with an aromatic substituent, was also obtained in high yield (59\%). Borylation of substrates (Z)-2g and $(Z)-\mathbf{2 h}$, with a branched structure at the $\varepsilon$ - and $\sigma$-positions, respectively, afforded the desired products in high yields $(63 \%$ and $72 \%$, respectively). However, $(Z)-\mathbf{2} \mathbf{i}$, with a benzyl ether group at the $\sigma$-position, only gave a trace amount of product under the optimized reaction conditions. Finally, borylation of substrates with either a benzyl or silyl ether at the $\varepsilon$-position afforded the corresponding products $(E)-3 \mathbf{j}$ and $(E)$-3k in high yield (54\% and $66 \%$ yield, respectively). 
Scheme 3 Substrate Scope for the Copper(I)-Catalyzed Boryl Substitution of Allyl Aminals (Z)-2 ${ }^{a}$

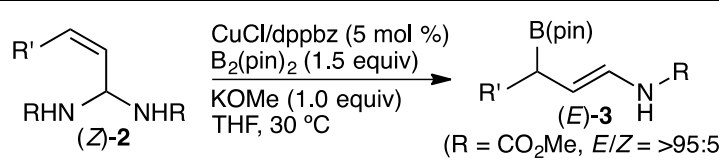

$$
\begin{aligned}
& (E)-3 d \\
& 3 \mathrm{~h}, 88 \%, E / Z=3: 1
\end{aligned}
$$<smiles>[R]N/C=C/C(Br)CCc1ccccc1</smiles><smiles>[R]N/C=C/C(Br)CC1CCCCC1</smiles><smiles>[R]N/C=C/C(Br)C1CC1</smiles><smiles>[R]N/C=C/C(Br)COCc1ccccc1</smiles><smiles>[R]N/C=C/C(Br)CCOCc1ccccc1</smiles><smiles>[R]N/C=C/C(Br)CCO[SH](=[18O])([18OH])[18OH]</smiles>

${ }^{a}$ Reagents and conditions: $\mathrm{CuCl}(0.0125 \mathrm{mmol})$, ligand $(0.0125 \mathrm{mmol}),(Z)-2(0.25$ $\mathrm{mmol})$, bis(pinacolato)diboron $(0.375 \mathrm{mmol})$ and base $(0.25 \mathrm{mmol})$ in solvent $(0.75$ $\mathrm{mL}$ ) at $30{ }^{\circ} \mathrm{C} .{ }^{b} 10 \mathrm{~mol} \%$ of $\mathrm{CuCl}$ and dppbz were used. $\mathrm{K}(\mathrm{O}-\mathrm{t}-\mathrm{Bu})$ was used instead of KOMe.

To demonstrate the usefulness of the $\gamma$ aminoallylboronate, we investigated the allylation of aldehydes (Table 2). We carried out the allylation of an aromatic aldehyde with (E)-3a to give the desired (Z)-anti-1,2-amino alcohol derivative in good yield and $E / Z$ selectivity (entry $1,82 \%$ yield, $E / Z=17: 83)$. The allylations of an aliphatic or conjugated aldehyde also proceeded smoothly to afford the corresponding products in good yields (entry $2,84 \%$ yield, $E / Z=23: 77$; entry 3 $91 \%$ yield, $E / Z=19: 81$, respectively). The use of Cbz-protected $\gamma$-aminoallylboronate $(E)$-3c displayed good yield and $E / Z$ selectivity in the aldehyde allylation (entry $4,76 \%$ yield, $E / Z=$ 17:83). Allylation with (E)-3j, containing a benzyl ether, afforded the corresponding (Z)-anti-1,2-amino alcohol in good yield with moderate $E / Z$ selectivity ( $84 \%$ yield, $E / Z=27: 73$ ).

Table 2 Aldehyde Allylation with $\gamma$-Aminoallylboronates $(E)-3^{a}$.

\begin{tabular}{|c|c|l|c|c|}
\hline \multicolumn{5}{|c|}{ (Z)-anti-4 } \\
\hline Entry & Substrate & \multicolumn{1}{|c|}{$\mathrm{RCHO}$} & Yield (\%) \\
\hline 1 & $(Z)$-3a & 4-nitrobenzaldehyde & $17: 83$ & 82 \\
\hline 2 & $(Z)-3 a$ & 3-phenylpropionaldehyde & $23: 77$ & 84 \\
\hline 3 & $(Z)-3 a$ & cinnamaldehyde & $19: 81$ & 91 \\
\hline 4 & $(Z)-3 c$ & 4-nitrobenzaldehyde & $17: 83$ & 76 \\
\hline 5 & $(Z)-3 j$ & 4-nitrobenzaldehyde & $27: 73$ & 84 \\
\hline
\end{tabular}

$a$ Reagents and conditions: $(E)-3(0.15-0.2 \mathrm{mmol})$, aldehydes $(0.45-0.6 \mathrm{mmol})$ in toluene at $50{ }^{\circ} \mathrm{C}$. The $E / Z$ ratios of the anti product were determined by ${ }^{1} \mathrm{H}$ NMR and HPLC analyses.
In summary, we have successfully developed a novel approach for the synthesis of linear $\gamma$-aminoallylboronates via copper(I)-catalyzed boryl substitution of allyl aminals. Allylation of aldehydes has been demonstrated with the synthesized boronates to afford (Z)-anti-1,2-amino alcohol derivatives in high yield and with good $E / Z$ selectivity. The borylation/allylation procedure is a concise and straightforward approach to construct a vicinal amino alcohol unit with concomitant formation of a new $\mathrm{C}-\mathrm{C}$ bond.

Materials were obtained from commercial suppliers and purified by standard procedures unless otherwise noted. Solvents were also purchased from commercial suppliers, distilled, degassed via three freeze-pump-thaw cycles, and further dried over molecular sieve (MS 4Å). NMR spectra were recorded on JEOL JNM-ECX400P and ECS-400 spectrometer $\left({ }^{1} \mathrm{H}: 400 \mathrm{MHz}\right.$ and ${ }^{13} \mathrm{C}$ : $\left.99 \mathrm{MHz}\right)$. Tetramethylsilane $\left({ }^{1} \mathrm{H}\right)$ and $\mathrm{CDCl}_{3}\left({ }^{13} \mathrm{C}\right)$ were employed as external standards, respectively. Multiplicity was recorded as follows: $s=$ singlet, brs = broad singlet, $d=$ doublet, $\mathrm{t}=$ triplet, $\mathrm{q}=$ quartet, quint = quintet, sept = septet, $\mathrm{o}=$ octet, $\mathrm{m}$ $=$ multiplet. $\mathrm{CuCl}$ (ReagentPlus ${ }^{\circledR}$ grade, 224332-25G, $\geq 99 \%$ ) was purchased from Sigma-Aldrich Co. Mesitylene and 1,4diisopropylbenzene were used as internal standards to determine NMR and GC yield, respectively. GLC analyses were conducted with a Shimadzu GC-2014 or GC-2025 equipped with ULBON HR-1 glass capillary column (Shinwa Chemical Industries) and a FID detector. HPLC analyses with chiral stationary phase were carried out using a Hitachi LaChrome Elite HPLC system with a L-2400 UV detector. High-resolution mass spectra were recorded at the Instrumental Analysis Division in Global Facility Center, Hokkaido University.

\section{A representative procedure for the copper(I)-catalyzed boryl substitution of $(Z)-2$ a (Table 1$)$}

CuCl (1.2 mg, $0.0125 \mathrm{mmol}), \mathrm{dppbz}(5.5 \mathrm{mg}, 0.0125 \mathrm{mmol})$, bis(pinacolato)diboron ( $95.2 \mathrm{mg}, 0.375 \mathrm{mmol}$ ) and KOMe $(17.5 \mathrm{mg}, 0.25$ mmol) were placed in a screw-capped test tube. The test tube was connected to a vacuum/nitrogen manifold through a needle. It was evacuated and backfilled with $\mathrm{N}_{2}$ gas three times. Then, dry THF $(0.5 \mathrm{ml})$ was added to the mixture via a syringe with stirring at $30 \stackrel{\circ}{\circ}$. After 15-30 min, aminal (Z)-2a (64.5 $\mathrm{mg}, 0.25 \mathrm{mmol}$ ) was added to the reaction mixture with vigorous stirring at $30^{\circ} \mathrm{C}$. After the completion of the reaction, the mixture was directly filtered through a short silica-gel column with $\mathrm{Et}_{2} \mathrm{O}$ as the eluent. After removal of the solvents under reduced pressure, GC yield was determined by GLC analysis of the crude reaction mixture $[(E)-3 a ; 89 \%]$ using 1,4-diisopropylbenzene $(26.7 \mathrm{mg}$, $0.164 \mathrm{mmol}$ ) as the internal standard. The crude product was purified by flash chromatography $\left(\mathrm{SiO}_{2}\right.$, hexane/AcOEt $=90: 10$ to 80:20) to give the corresponding $\gamma$-aminoallylboronate $(E)$-3a (55.1 mg, $0.177 \mathrm{mmol}, 71 \%$ isolated yield).

A representative procedure for preparation of $(Z)$-dimethyl (oct-2ene-1,1-diyl)dicarbamate $[(Z)-2 a]$

In an oven-dried $30 \mathrm{~mL}$ two-neck round-bottomed flask, octynal (4.28 $\mathrm{mL}, 30 \mathrm{mmol})$, methylcarbamate $(3.60 \mathrm{~g}, 48 \mathrm{mmol})$ and acetic anhydride $(7.0 \mathrm{~mL})$ were charged under nitrogen atmosphere. Trifluoroacetic acid $(0.2 \mathrm{~mL})$ was added to the suspension and the mixture was stirred for 15 min. The product was solidified in acetic anhydride solvent as a white solid. The solid was filtered in vacuum, washed with hexane and purified by column chromatography $\left(\mathrm{SiO}_{2}\right.$, hexane/AcOEt $=70: 30$ to $\left.40: 60\right)$ to give the product as a white powder ( $3.53 \mathrm{~g}, 46 \%$ yield).

In screw-capped test tube, $\mathrm{IPrCuCl}(48.7 \mathrm{mg}, 0.1 \mathrm{mmol})$ and $\mathrm{K}(\mathrm{O}-\mathrm{t}$-Bu$)$ (11.2 $\mathrm{mg}, 0.1 \mathrm{mmol}$ ) were dissolved in dry THF (1.2 mL) under a nitrogen atmosphere. The catalyst solution was allowed to stir vigorously for $20 \mathrm{~min}$ and added to a solution of the propargyl aminal (530.7 mg, $2.1 \mathrm{mmol})$, 1,1,3,3-tetramethyldisiloxane $(742.3 \mu \mathrm{L}, 4.2$ mmol), $t$-BuOH $(505.4 \mu \mathrm{L}, 5.25 \mathrm{mmol})$ and THF $(3.0 \mathrm{~mL})$. The mixture was directly filtered through a short silica-gel column with $\mathrm{Et}_{2} \mathrm{O}$ as the eluent. After removal of the solvents under reduced pressure, the crude product was purified by flash chromatography $\left(\mathrm{SiO}_{2}\right.$, 
hexane $\left./ \mathrm{AcOEt} / \mathrm{CH}_{2} \mathrm{Cl}_{2}=50: 30: 20\right)$ to give the product $(Z)-\mathbf{2 a}(271.4 \mathrm{mg}$, $51 \%$ yield).

${ }^{1} \mathrm{H}$ NMR (401 MHz, $\left.\mathrm{CDCl}_{3}, \delta\right): 0.88(\mathrm{t}, J=6.8 \mathrm{~Hz}, 3 \mathrm{H}), 1.18-1.42(\mathrm{~m}, 6 \mathrm{H})$ $2.13(\mathrm{q}, J=7.1 \mathrm{~Hz}, 2 \mathrm{H}), 3.68(\mathrm{~s}, 6 \mathrm{H}), 5.30-5.90(\mathrm{~m}, 4 \mathrm{H}), 5.55(\mathrm{dt}, J=7.4$ $9.6 \mathrm{~Hz}, 1 \mathrm{H})$.

${ }^{13} \mathrm{C}$ NMR (100 MHz, $\left.\mathrm{CDCl}_{3}, \delta\right): 13.9\left(\mathrm{CH}_{3}\right), 22.4\left(\mathrm{CH}_{2}\right), 27.6\left(\mathrm{CH}_{2}\right), 28.8$ $\left(\mathrm{CH}_{2}\right), 31.3\left(\mathrm{CH}_{2}\right), 52.1\left(\mathrm{CH}_{3}\right), 56.8(\mathrm{CH}), 126.3(\mathrm{CH}), 133.9(\mathrm{CH}), 155.9(\mathrm{C})$ HRMS-ESI $(m / z):[\mathrm{M}+\mathrm{Na}]^{+}$calcd for $\mathrm{C}_{12} \mathrm{H}_{22} \mathrm{O}_{4} \mathrm{~N}_{2} \mathrm{Na}, 281.14718$; found, 281.14719.

\section{(Z)-Di-tert-butyl (oct-2-ene-1,1-diyl)dicarbamate [(Z)-2b]}

${ }^{1} \mathrm{H}$ NMR (401 MHz, $\left.\mathrm{CDCl}_{3}, \delta\right): 0.89(\mathrm{t}, J=6.8 \mathrm{~Hz}, 3 \mathrm{H}), 1.19-1.41(\mathrm{~m}, 6 \mathrm{H})$ $1.44(\mathrm{~s}, 18 \mathrm{H}), 2.12(\mathrm{q}, J=6.8 \mathrm{~Hz}, 2 \mathrm{H}), 5.37(\mathrm{br} \mathrm{s}, 2 \mathrm{H}), 5.50(\mathrm{dt}, J=7.5,9.8$ $\mathrm{Hz}, 1 \mathrm{H}), 5.45-5.65(\mathrm{~m}, 1 \mathrm{H}), 5.55-5.90(\mathrm{~m}, 1 \mathrm{H})$.

${ }^{13} \mathrm{C}$ NMR (100 MHz, $\left.\mathrm{CDCl}_{3}, \delta\right): 13.8\left(\mathrm{CH}_{3}\right), 22.4\left(\mathrm{CH}_{2}\right), 27.5\left(\mathrm{CH}_{2}\right), 28.2$ $\left(\mathrm{CH}_{3}\right), 28.8\left(\mathrm{CH}_{2}\right), 31.2\left(\mathrm{CH}_{2}\right), 56.2(\mathrm{CH}), 79.4(\mathrm{C}), 127.3(\mathrm{CH}), 132.8(\mathrm{CH})$, $154.7(C)$.

HRMS-ESI $(m / z):[M+N a]^{+}$calcd for $\mathrm{C}_{18} \mathrm{H}_{34} \mathrm{O}_{4} \mathrm{~N}_{2} \mathrm{Na}$, 365.24108; found, 365.24103 .

\section{(Z)-Dibenzyl (oct-2-ene-1,1-diyl)dicarbamate [(Z)-2c]}

${ }^{1} \mathrm{H}$ NMR (401 MHz, $\left.\mathrm{CDCl}_{3}, \delta\right): 0.87(\mathrm{t}, J=7.0 \mathrm{~Hz}, 3 \mathrm{H}), 1.19-1.41(\mathrm{~m}, 6 \mathrm{H})$ $2.11(\mathrm{q}, J=7.0 \mathrm{~Hz}, 2 \mathrm{H}), 5.10(\mathrm{~s}, 4 \mathrm{H}), 5.55(\mathrm{dt}, J=7.8,9.6 \mathrm{~Hz}, 1 \mathrm{H}), 5.51-$ $5.92(\mathrm{~m}, 4 \mathrm{H}), 7.27-7.40(\mathrm{~m}, 10 \mathrm{H})$.

${ }^{13} \mathrm{C}$ NMR $\left(100 \mathrm{MHz}, \mathrm{CDCl}_{3}, \delta\right): 13.9\left(\mathrm{CH}_{3}\right), 22.4\left(\mathrm{CH}_{2}\right), 27.6\left(\mathrm{CH}_{2}\right), 28.8$ $\left(\mathrm{CH}_{2}\right), 31.2\left(\mathrm{CH}_{2}\right), 56.8(\mathrm{CH}), 66.7\left(\mathrm{CH}_{2}\right), 126.2(\mathrm{CH}), 127.96(\mathrm{CH}), 128.02$ $(\mathrm{CH}), 128.4(\mathrm{CH}), 133.9(\mathrm{CH}), 136.1(\mathrm{C}), 155.2(\mathrm{C})$.

HRMS-ESI $(\mathrm{m} / z)$ : [M+Na] ${ }^{+}$calcd for $\mathrm{C}_{24} \mathrm{H}_{30} \mathrm{O}_{4} \mathrm{~N}_{2} \mathrm{Na}, 433.20978$; found, 433.20981 .

\section{Dimethyl (prop-2-ene-1,1-diyl)dicarbamate (2d)}

${ }^{1} \mathrm{H}$ NMR (401 MHz, $\left.\mathrm{CDCl}_{3}, \delta\right): 3.69(\mathrm{~s}, 6 \mathrm{H}), 5.25(\mathrm{~d}, J=10.4 \mathrm{~Hz}, 1 \mathrm{H}), 5.34$ (d, $J=17.6 \mathrm{~Hz}, 1 \mathrm{H}), 5.40-5.88(\mathrm{~m}, 3 \mathrm{H}), 5.89-6.10(\mathrm{~m}, 1 \mathrm{H})$.

${ }^{13} \mathrm{C}$ NMR (100 MHz, $\left.\mathrm{CDCl}_{3}, \delta\right): 52.3\left(\mathrm{CH}_{3}\right), 60.6(\mathrm{CH}), 116.7\left(\mathrm{CH}_{2}\right), 135.2$ $(\mathrm{CH}), 156.0(\mathrm{C})$.

HRMS-ESI $(m / z)$ : $[\mathrm{M}+\mathrm{Na}]^{+}$calcd for $\mathrm{C}_{7} \mathrm{H}_{12} \mathrm{O}_{4} \mathrm{~N}_{2} \mathrm{Na}, 211.06893$; found, 211.06932

\section{(Z)-Dimethyl (but-2-ene-1,1-diyl)dicarbamate [(Z)-2e]}

${ }^{1} \mathrm{H}$ NMR (401 MHz, $\left.\mathrm{CDCl}_{3}, \delta\right): 1.72(\mathrm{~d}, J=5.6 \mathrm{~Hz}, 3 \mathrm{H}), 3.68(\mathrm{~s}, 6 \mathrm{H}), 5.63(\mathrm{dt}$ $J=7.1,9.9 \mathrm{~Hz}, 1 \mathrm{H}), 5.15-6.00(\mathrm{~m}, 4 \mathrm{H})$

${ }^{13} \mathrm{C}$ NMR (100 MHz, $\left.\mathrm{CDCl}_{3}, \delta\right): 13.3\left(\mathrm{CH}_{3}\right), 52.2\left(\mathrm{CH}_{3}\right), 56.6(\mathrm{CH}), 127.4$ $(\mathrm{CH}), 128.0(\mathrm{CH}), 156.0(\mathrm{C})$.

HRMS-ESI $(m / z):[\mathrm{M}+\mathrm{Na}]^{+}$calcd for $\mathrm{C}_{8} \mathrm{H}_{14} \mathrm{O}_{4} \mathrm{~N}_{2} \mathrm{Na}, 225.08458$; found, 225.08465 .

\section{(Z)-Dibenzyl (oct-2-ene-1,1-diyl)dicarbamate [(Z)-2f]}

${ }^{1} \mathrm{H}$ NMR (400 MHz, $\mathrm{CDCl}_{3}, \delta$ ): $2.47(\mathrm{q}, J=7.1 \mathrm{~Hz}, 2 \mathrm{H}$ ), $2.68(\mathrm{t}, J=7.6 \mathrm{~Hz}$, $2 \mathrm{H}), 3.64(\mathrm{~s}, 6 \mathrm{H}), 5.55(\mathrm{dt}, J=7.6,10.6 \mathrm{~Hz}, 1 \mathrm{H}), 5.20-5.90(\mathrm{~m}, 4 \mathrm{H}), 7.15-$ $7.22(\mathrm{~m}, 3 \mathrm{H}), 7.23-7.31(\mathrm{~m}, 2 \mathrm{H})$.

${ }^{13} \mathrm{C}$ NMR (100 MHz, $\left.\mathrm{CDCl}_{3}, \delta\right): 29.5\left(\mathrm{CH}_{2}\right), 35.3\left(\mathrm{CH}_{2}\right), 52.1\left(\mathrm{CH}_{3}\right), 56.8$ $(\mathrm{CH}), 125.9(\mathrm{CH}), 127.0(\mathrm{CH}), 128.3(\mathrm{CH}), 128.5(\mathrm{CH}), 132.2(\mathrm{CH}), 141.2$ (C), $155.9(C)$.

HRMS-ESI $(m / z)$ : $[\mathrm{M}+\mathrm{Na}]^{+}$calcd for $\mathrm{C}_{15} \mathrm{H}_{20} \mathrm{O}_{4} \mathrm{~N}_{2} \mathrm{Na}, 315.13153$; found, 315.13132 .

(Z)-Dimethyl (4-cyclohexylbut-2-ene-1,1-diyl)dicarbamate [(Z)-2g] ${ }^{1} \mathrm{H}$ NMR (401 MHz, $\left.\mathrm{CDCl}_{3}, \delta\right): 0.81-1.11(\mathrm{~m}, 2 \mathrm{H}), 1.15-1.38(\mathrm{~m}, 4 \mathrm{H}), 1.58-$ $1.74(\mathrm{~m}, 5 \mathrm{H}), 2.01(\mathrm{t}, J=6.6 \mathrm{~Hz}, 2 \mathrm{H}), 3.68(\mathrm{~s}, 6 \mathrm{H}), 5.56(\mathrm{dt}, J=7.7,10.4 \mathrm{~Hz}$, $1 \mathrm{H}), 5.29-5.91(\mathrm{~m}, 4 \mathrm{H})$.

${ }^{13} \mathrm{C}$ NMR (101 MHz, $\left.\mathrm{CDCl}_{3}, \delta\right): 26.1\left(\mathrm{CH}_{2}\right), 26.2\left(\mathrm{CH}_{2}\right), 32.8\left(\mathrm{CH}_{2}\right), 35.2$ $\left(\mathrm{CH}_{2}\right), 37.7\left(\mathrm{CH}_{3}\right), 51.9(\mathrm{CH}), 56.6(\mathrm{CH}), 126.8(\mathrm{CH}), 131.9(\mathrm{CH}), 155.9(\mathrm{C})$. HRMS-ESI $(m / z):[M+N a]^{+}$calcd for $\mathrm{C}_{14} \mathrm{H}_{24} \mathrm{O}_{4} \mathrm{~N}_{2} \mathrm{Na}, 307.16283$; found, 307.16263 .
(Z)-Dimethyl (3-cyclopropylprop-2-ene-1,1-diyl)dicarbamate [(Z)2h]

$\left.{ }^{1} \mathrm{H} \mathrm{NMR} \mathrm{(401} \mathrm{MHz,} \mathrm{CDCl}_{3}, \delta\right): 0.40(\mathrm{dt}, J=4.5,6.6 \mathrm{~Hz}, 2 \mathrm{H}), 0.82(\mathrm{dt}, J=4.4$, $6.4 \mathrm{~Hz}, 2 \mathrm{H}), 1.53-1.67(\mathrm{~m}, 1 \mathrm{H}), 3.69(\mathrm{~s}, 6 \mathrm{H}), 4.89(\mathrm{t}, J=10.2 \mathrm{~Hz}, 1 \mathrm{H})$, 5.20-5.78 (m, 3H), $5.87(\mathrm{q}, J=7.2 \mathrm{~Hz}, 1 \mathrm{H})$.

${ }^{13} \mathrm{C}$ NMR (100 MHz, $\left.\mathrm{CDCl}_{3}, \delta\right): 7.3\left(\mathrm{CH}_{2}\right), 10.0(\mathrm{CH}), 52.2\left(\mathrm{CH}_{3}\right), 57.3(\mathrm{CH})$, $124.3(\mathrm{CH}), 137.9(\mathrm{CH}), 156.0(\mathrm{C})$.

HRMS-ESI $(m / z):[\mathrm{M}+\mathrm{Na}]^{+}$calcd for $\mathrm{C}_{10} \mathrm{H}_{16} \mathrm{O}_{4} \mathrm{~N}_{2} \mathrm{Na}, 251.10023$; found, 251.10025 .

(Z)-Dimethyl (4-(benzyloxy)but-2-ene-1,1-diyl)dicarbamate [(Z)-2i] ${ }^{1} \mathrm{H}$ NMR (401 MHz, $\left.\mathrm{CDCl}_{3}, \delta\right): 3.67(\mathrm{~s}, 6 \mathrm{H}), 4.15(\mathrm{dd}, J=1.2,6.0 \mathrm{~Hz}, 2 \mathrm{H})$, $4.52(\mathrm{~s}, 2 \mathrm{H}), 5.73(\mathrm{dt}, J=6.0,17.0 \mathrm{~Hz}, 1 \mathrm{H}), 5.35-5.80(\mathrm{~m}, 2 \mathrm{H}), 5.81-6.05$ $(\mathrm{m}, 2 \mathrm{H}), 7.26-7.40(\mathrm{~m}, 5 \mathrm{H})$.

${ }^{13} \mathrm{C}$ NMR (100 MHz, $\left.\mathrm{CDCl}_{3}, \delta\right): 52.0\left(\mathrm{CH}_{3}\right), 56.7(\mathrm{CH}), 65.7\left(\mathrm{CH}_{2}\right), 72.3$ $\left(\mathrm{CH}_{2}\right), 127.5(\mathrm{CH}), 127.6(\mathrm{CH}), 128.2(\mathrm{CH}), 129.1(\mathrm{CH}), 129.2(\mathrm{CH}), 137.7$ (C), $155.8(C)$

HRMS-ESI $(m / z)$ : $[\mathrm{M}+\mathrm{Na}]^{+}$calcd for $\mathrm{C}_{15} \mathrm{H}_{20} \mathrm{O}_{5} \mathrm{~N}_{2} \mathrm{Na}, 331.12644$; found, 331.12625

(Z)-Dimethyl (5-(benzyloxy)pent-2-ene-1,1-diyl)dicarbamate [(Z)2j]

${ }^{1} \mathrm{H}$ NMR (401 MHz, $\left.\mathrm{CDCl}_{3}, \delta\right): 2.47(\mathrm{q}, J=6.4 \mathrm{~Hz}, 2 \mathrm{H}), 3.50(\mathrm{t}, J=6.4 \mathrm{~Hz}$, $2 \mathrm{H}), 3.67(\mathrm{~s}, 6 \mathrm{H}), 4.94(\mathrm{~s}, 2 \mathrm{H}), 5.60(\mathrm{dt}, J=7.5,10.0 \mathrm{~Hz}, 1 \mathrm{H}), 5.65-5.88(\mathrm{~m}$, $2 \mathrm{H}), 5.89-6.28(\mathrm{~m}, 2 \mathrm{H}), 7.22-7.38(\mathrm{~m}, 5 \mathrm{H})$.

${ }^{13} \mathrm{C}$ NMR (100 MHz, $\left.\mathrm{CDCl}_{3}, \delta\right): 28.0\left(\mathrm{CH}_{2}\right), 51.9\left(\mathrm{CH}_{3}\right), 56.6(\mathrm{CH}), 68.9$ $\left(\mathrm{CH}_{2}\right), 72.5\left(\mathrm{CH}_{2}\right), 127.3(\mathrm{CH}), 127.4(\mathrm{CH}), 128.1(\mathrm{CH}), 128.3(\mathrm{CH}), 129.4$ $(\mathrm{CH}), 138.0(C), 155.8(C)$.

HRMS-ESI $(m / z):[M+N a]^{+}$calcd for $\mathrm{C}_{16} \mathrm{H}_{22} \mathrm{O}_{5} \mathrm{~N}_{2} \mathrm{Na}, 345.14209$; found, 345.14182 .

(Z)-Dimethyl $\quad\{5-[($ tert-butyldiphenylsilyl)oxy]pent-2-ene-1,1diyl\}dicarbamate $[(Z)-2 \mathrm{k}]$

${ }^{1} \mathrm{H}$ NMR (401 MHz, $\left.\mathrm{CDCl}_{3}, \delta\right): 1.04(\mathrm{~s}, 9 \mathrm{H}), 2.40(\mathrm{q}, J=6.3 \mathrm{~Hz}, 2 \mathrm{H}), 3.66(\mathrm{~s}$, $6 \mathrm{H}), 3.68(\mathrm{t}, J=6.4 \mathrm{~Hz}, 2 \mathrm{H}), 5.20-5.70(\mathrm{~m}, 2 \mathrm{H}), 5.61(\mathrm{dt}, J=7.4,10.7 \mathrm{~Hz}$, 1H), 5.70-5.98 (m, 2H), 7.36-7.48 (m, 6H), 7.62-7.70 (m, 4H).

${ }^{13} \mathrm{C}$ NMR (101 MHz, $\left.\mathrm{CDCl}_{3}, \delta\right): 19.1(\mathrm{C}), 26.7\left(\mathrm{CH}_{3}\right), 30.9\left(\mathrm{CH}_{2}\right), 52.0\left(\mathrm{CH}_{3}\right)$, $56.8(\mathrm{CH}), 62.9\left(\mathrm{CH}_{2}\right), 127.5(\mathrm{CH}), 128.0(\mathrm{CH}), 129.5(\mathrm{CH}), 129.9(\mathrm{CH})$, $133.5(\mathrm{C}), 135.4(\mathrm{CH}), 155.9(\mathrm{C})$.

HRMS-ESI $(\mathrm{m} / \mathrm{z})$ : $[\mathrm{M}+\mathrm{Na}]^{+}$calcd for $\mathrm{C}_{25} \mathrm{H}_{34} \mathrm{O}_{5} \mathrm{~N}_{2} \mathrm{NaSi}, 493.21292$; found, 493.21277.

Methyl (E)-[3-(4,4,5,5-tetramethyl-1,3,2-dioxaborolan-2-yl)oct-1en-1-yl]carbamate $[(E)-3 a]$

${ }^{1} \mathrm{H}$ NMR (401 MHz, $\left.\mathrm{CDCl}_{3}, \delta\right): 0.87(\mathrm{t}, J=6.6 \mathrm{~Hz}, 3 \mathrm{H}), 1.19-1.41(\mathrm{~m}, 7 \mathrm{H})$, $1.23(\mathrm{~s}, 12 \mathrm{H}), 1.44-1.54(\mathrm{~m}, 1 \mathrm{H}), 1.64-1.77(\mathrm{~m}, 1 \mathrm{H}), 3.69(\mathrm{~s}, 3 \mathrm{H}), 4.96(\mathrm{dd}$ $J=9.2,14.4 \mathrm{~Hz}, 1 \mathrm{H}), 6.19(\mathrm{~d}, J=10.4 \mathrm{~Hz}, 1 \mathrm{H}), 6.43(\mathrm{dd}, J=10.6,13.8 \mathrm{~Hz}$, $1 \mathrm{H})$.

${ }^{13} \mathrm{C}$ NMR (100 MHz, $\left.\mathrm{CDCl}_{3}, \delta\right): 14.0\left(\mathrm{CH}_{3}\right), 22.5\left(\mathrm{CH}_{2}\right), 24.58\left(\mathrm{CH}_{3}\right), 24.64$ $\left(\mathrm{CH}_{3}\right), 25.3$ (br, B-CH), $28.5\left(\mathrm{CH}_{2}\right), 31.2\left(\mathrm{CH}_{2}\right), 31.8\left(\mathrm{CH}_{2}\right), 52.2\left(\mathrm{CH}_{3}\right), 83.1$ $(C), 111.7(\mathrm{CH}), 122.8(\mathrm{CH}), 154.1(\mathrm{C})$.

HRMS-ESI $(\mathrm{m} / \mathrm{z})$ : $[\mathrm{M}+\mathrm{Na}]^{+}$calcd for $\mathrm{C}_{16} \mathrm{H}_{30} \mathrm{O}_{4} \mathrm{BNa}, 333.21964$; found, 333.22026

Methyl (E)-[3-(4,4,5,5-tetramethyl-1,3,2-dioxaborolan-2-yl)prop-1en-1-yl]carbamate [(E)-3d]

${ }^{1} \mathrm{H}$ NMR (400 MHz, $\mathrm{CDCl}_{3}$, * indicates signals of the minor $Z$-isomer, $\delta$ ): $1.248(\mathrm{~s}, 12 \mathrm{H}), 1.254^{*}(\mathrm{~s}, 12 \mathrm{H}), 1.54^{*}(\mathrm{~d}, J=8.0 \mathrm{~Hz}, 2 \mathrm{H}), 1.62(\mathrm{~d}, J=7.6 \mathrm{~Hz}$ $2 \mathrm{H}), 3.69(\mathrm{~s}, 3 \mathrm{H}), 3.73^{*}(\mathrm{~s}, 3 \mathrm{H}), 4.74^{*}(\mathrm{q}, J=8.0 \mathrm{~Hz}, 1 \mathrm{H}), 5.04(\mathrm{dt}, J=7.2$, $14.3 \mathrm{~Hz}, 1 \mathrm{H}), 6.21(\mathrm{~d}, J=8.4 \mathrm{~Hz}, 1 \mathrm{H}), 6.44(\mathrm{t}, J=12.4 \mathrm{~Hz}, 1 \mathrm{H})$.

${ }^{13} \mathrm{C}$ NMR (100 MHz, $\mathrm{CDCl}_{3}$, * indicates signals of the minor $Z$-isomer, $\delta$ ): $12.4\left(\mathrm{~B}-\mathrm{CH}_{2}\right), 24.6^{*}\left(\mathrm{CH}_{3}\right), 24.7\left(\mathrm{CH}_{3}\right), 52.2\left(\mathrm{CH}_{3}\right), 52.3^{*}\left(\mathrm{CH}_{3}\right), 83.3(\mathrm{C})$, 103.3* $(\mathrm{CH}), 105.9(\mathrm{CH}), 122.9^{*}(\mathrm{CH}), 123.6(\mathrm{CH}), 154.1(\mathrm{C}), 154.4^{*}(\mathrm{C})$. The carbon directly attached to the boron atom was not detected, likely due to quadrupolar relaxation. 
HRMS-ESI $(\mathrm{m} / \mathrm{z})$ : [M+Na] ${ }^{+}$calcd for $\mathrm{C}_{11} \mathrm{H}_{20} \mathrm{O}_{4} \mathrm{NBNa}, 263.14139$; found, 263.14176

Methyl (E)-[3-(4,4,5,5-tetramethyl-1,3,2-dioxaborolan-2-yl)but-1en-1-yl]carbamate $[(E)-3 e]$

${ }^{1} \mathrm{H}$ NMR (401 MHz, $\mathrm{CDCl}_{3}$, only major isomer is shown, $\delta$ ): $1.09(\mathrm{~d}, J=7.2$ $\mathrm{Hz}, 3 \mathrm{H}), 1.21(\mathrm{~s}, 12 \mathrm{H}), 1.74-1.88(\mathrm{~m}, 1 \mathrm{H}), 3.69(\mathrm{~s}, 3 \mathrm{H}), 5.12(\mathrm{q}, J=7.4 \mathrm{~Hz}$ 1H), 6.10-6.28 (m, 1H), $6.42(\mathrm{dd}, J=11.6,13.2 \mathrm{~Hz}, 1 \mathrm{H})$.

${ }^{13} \mathrm{C}$ NMR (100 MHz, $\left.\mathrm{CDCl}_{3}, \delta\right): 15.5\left(\mathrm{CH}_{3}\right), 18.4(\mathrm{br}, \mathrm{B}-\mathrm{CH}), 24.6\left(\mathrm{CH}_{3}\right)$ $52.2\left(\mathrm{CH}_{3}\right), 83.2(\mathrm{C}), 113.2(\mathrm{CH}), 122.0(\mathrm{CH}), 154.1(\mathrm{C})$.

HRMS-ESI $(\mathrm{m} / \mathrm{z})$ : $[\mathrm{M}+\mathrm{Na}]^{+}$calcd for $\mathrm{C}_{12} \mathrm{H}_{22} \mathrm{O}_{4} \mathrm{NBNa}, 277.15704$; found, 277.15732

Methyl (E)-[5-phenyl-3-(4,4,5,5-tetramethyl-1,3,2-dioxaborolan-2yl)]pent-1-en-1-ylcarbamate [(E)-3f]

${ }^{1} \mathrm{H}$ NMR (400 MHz, $\left.\mathrm{CDCl}_{3}, \delta\right): 1.24(\mathrm{~s}, 12 \mathrm{H}), 1.60-1.72(\mathrm{~m}, 1 \mathrm{H}), 1.73-1.91$ (m, 2H), 2.50-2.70 (m, 2H), $3.70(\mathrm{~s}, 3 \mathrm{H}), 5.00(\mathrm{dd}, J=9.2,14.0 \mathrm{~Hz}, 1 \mathrm{H})$ $6.22(\mathrm{~d}, J=10.8 \mathrm{~Hz}, 1 \mathrm{H}), 6.47(\mathrm{dd}, J=10.8,13.6 \mathrm{~Hz}, 1 \mathrm{H}), 7.17(\mathrm{~d}, J=6.4 \mathrm{~Hz}$ $3 \mathrm{H}), 7.22-7.30(\mathrm{~m}, 2 \mathrm{H})$

${ }^{13} \mathrm{C}$ NMR (100 MHz, $\left.\mathrm{CDCl}_{3}, \delta\right): 24.6\left(\mathrm{CH}_{3}\right), 24.7\left(\mathrm{CH}_{3}\right), 24.9(\mathrm{br}, \mathrm{B}-\mathrm{CH})$, $33.2\left(\mathrm{CH}_{2}\right), 35.1\left(\mathrm{CH}_{2}\right), 52.2\left(\mathrm{CH}_{3}\right), 83.2(\mathrm{C}), 111.1(\mathrm{CH}), 123.3(\mathrm{CH}), 125.6$ $(\mathrm{CH}), 128.2(\mathrm{CH}), 128.4(\mathrm{CH}), 142.5(\mathrm{C}), 154.0(\mathrm{C})$.

HRMS-ESI $(\mathrm{m} / \mathrm{z})$ : [M+Na] ${ }^{+}$calcd for $\mathrm{C}_{19} \mathrm{H}_{28} \mathrm{O}_{4} \mathrm{NBNa}, 367.20399$; found, 367.20465 .

Methyl

(E)-[4-cyclohexyl-3-(4,4,5,5-tetramethyl-1,3,2 dioxaborolan-2-yl)but-1-en-1-yl]carbamate [(E)-3g]

${ }^{1} \mathrm{H}$ NMR (399 MHz, $\left.\mathrm{CDCl}_{3}, \delta\right): 0.70-0.94(\mathrm{~m}, 2 \mathrm{H}), 1.04-1.44(\mathrm{~m}, 7 \mathrm{H}), 1.23$ (s, 12H), 1.57-1.76 (m, 4H), 1.76-1.92 (m, 1H), $3.69(\mathrm{~s}, 3 \mathrm{H}), 4.92$ (dd, $J=$ 9.0, 14.2 Hz, 1H), 6.18 (d, $J=10.4 \mathrm{~Hz}, 1 \mathrm{H}), 6.42$ (dd, $J=11.0,14.2 \mathrm{~Hz}, 1 \mathrm{H}$ ).

${ }^{13} \mathrm{C}$ NMR (100 MHz, $\left.\mathrm{CDCl}_{3}, \delta\right): 22.4(\mathrm{br}, \mathrm{B}-\mathrm{CH}), 24.6\left(\mathrm{CH}_{3}\right), 26.2\left(\mathrm{CH}_{2}\right)$, $26.3\left(\mathrm{CH}_{2}\right), 26.6\left(\mathrm{CH}_{2}\right), 32.7\left(\mathrm{CH}_{2}\right), 33.6\left(\mathrm{CH}_{2}\right), 36.2(\mathrm{CH}), 38.7\left(\mathrm{CH}_{2}\right), 52.2$ $\left(\mathrm{CH}_{3}\right), 83.1(\mathrm{C}), 111.8(\mathrm{CH}), 122.6(\mathrm{CH}), 154.0(\mathrm{C})$.

HRMS-ESI $(m / z):[\mathrm{M}+\mathrm{Na}]^{+}$calcd for $\mathrm{C}_{18} \mathrm{H}_{32} \mathrm{O}_{4} \mathrm{NBNa}, 359.23529$; found, 359.23615

\section{Methyl (E)-[3-cyclopropyl-3-(4,4,5,5-tetramethyl-1,3,2-} dioxaborolan-2-yl)prop-1-en-1-yl]carbamate $[(E)-3 \mathrm{~h}]$

${ }^{1} \mathrm{H}$ NMR (399 MHz, $\left.\mathrm{CDCl}_{3}, \delta\right): 0.02-0.10(\mathrm{~m}, 1 \mathrm{H}), 0.11-0.21(\mathrm{~m}, 1 \mathrm{H}), 0.44$ (d, $J=7.2 \mathrm{~Hz}, 2 \mathrm{H}), 0.76-0.85(\mathrm{~m}, 1 \mathrm{H}), 1.12-1.35(\mathrm{~m}, 1 \mathrm{H}), 1.25(\mathrm{~s}, 12 \mathrm{H})$ $3.70(\mathrm{~s}, 3 \mathrm{H}), 5.09$ (d, $J=7.5 \mathrm{~Hz}, 1 \mathrm{H}), 6.34(\mathrm{brs}, 1 \mathrm{H}), 6.50(\mathrm{t}, J=12.4 \mathrm{~Hz}$, $1 \mathrm{H})$.

${ }^{13} \mathrm{C}$ NMR $\left(100 \mathrm{MHz}, \mathrm{CDCl}_{3}, \delta\right): 3.8\left(\mathrm{CH}_{2}\right), 4.3\left(\mathrm{CH}_{2}\right), 12.2(\mathrm{CH}), 24.6\left(\mathrm{CH}_{3}\right)$ 29.5 (br, B-CH), $52.3\left(\mathrm{CH}_{3}\right), 83.2(\mathrm{C}), 110.8(\mathrm{CH}), 122.9(\mathrm{CH}), 154.1(\mathrm{C})$.

HRMS-ESI $(m / z):[M+N a]^{+}$calcd for $\mathrm{C}_{14} \mathrm{H}_{24} \mathrm{O}_{4} \mathrm{NBNa}, 303.17269$; found, 303.17313 .

\section{Methyl (E)-[5-(benzyloxy)-3-(4,4,5,5-tetramethyl-1,3,2 dioxaborolan-2-yl)pent-1-en-1-yl]carbamate [(E)-3j]}

${ }^{1} \mathrm{H}$ NMR (399 MHz, $\mathrm{CDCl}_{3}, \delta$ ): 1.20 (s, 12H), 1.59-1.74 (m, 1H), 1.80-1.94 (m, 2H), 3.38-3.52 (m, 2H), 3.69 (s, 3H), 4.48 (s, 2H), 4.97 (dd, $J=8.8$, $13.6 \mathrm{~Hz}, 1 \mathrm{H}), 6.26(\mathrm{~d}, J=9.2 \mathrm{~Hz}, 1 \mathrm{H}), 6.45(\mathrm{dd}, J=10.6,13.8 \mathrm{~Hz}, 1 \mathrm{H})$, 7.20-7.40 (m, 5H).

${ }^{13} \mathrm{C}$ NMR (100 MHz, $\left.\mathrm{CDCl}_{3}, \delta\right): 22.1(\mathrm{br}, \mathrm{B}-\mathrm{CH}), 24.6\left(\mathrm{CH}_{3}\right), 24.7\left(\mathrm{CH}_{3}\right)$, $31.2\left(\mathrm{CH}_{2}\right), 52.3\left(\mathrm{CH}_{3}\right), 69.2\left(\mathrm{CH}_{2}\right), 72.8\left(\mathrm{CH}_{2}\right), 83.2(\mathrm{C}), 110.9(\mathrm{CH}), 123.2$ $(\mathrm{CH}), 127.4(\mathrm{CH}), 127.7(\mathrm{CH}), 128.3(\mathrm{CH}), 138.6(\mathrm{C}), 154.0(\mathrm{C})$

HRMS-ESI $(\mathrm{m} / \mathrm{z})$ : $[\mathrm{M}+\mathrm{Na}]^{+}$calcd for $\mathrm{C}_{20} \mathrm{H}_{30} \mathrm{O}_{5} \mathrm{NBNa}, 397.21456$; found, 397.21508.

Methyl (E)-[5-((tert-butyldiphenylsilyl)oxy)-3-(4,4,5,5-tetramethyl1,3,2-dioxaborolan-2-yl)pent-1-en-1-yl]carbamate [(E)-3k]

${ }^{1} \mathrm{H}$ NMR (399 MHz, $\mathrm{CDCl}_{3}$, only major isomer is shown, $\left.\delta\right): 1.04(\mathrm{~s}, 9 \mathrm{H})$ $1.18(\mathrm{~s}, 6 \mathrm{H}), 1.19(\mathrm{~s}, 6 \mathrm{H}), 1.56-1.71(\mathrm{~m}, 1 \mathrm{H}), 1.84$ (sextet, $J=6.8 \mathrm{~Hz}, 1 \mathrm{H}$ ), $1.96(\mathrm{q}, J=8.0 \mathrm{~Hz}, 1 \mathrm{H}), 3.64(\mathrm{t}, J=6.6 \mathrm{~Hz}, 2 \mathrm{H}), 3.70(\mathrm{~s}, 3 \mathrm{H}), 4.89(\mathrm{dd}, J=$ 9.2, $14.0 \mathrm{~Hz}, 1 \mathrm{H}), 6.17(\mathrm{~d}, J=10.3 \mathrm{~Hz}, 1 \mathrm{H}), 6.43(\mathrm{dd}, J=10.8,14.0 \mathrm{~Hz}, 1 \mathrm{H})$ $7.36-7.46(\mathrm{~m}, 6 \mathrm{H}), 7.66(\mathrm{dd}, J=1.4,7.8 \mathrm{~Hz}, 4 \mathrm{H})$.
${ }^{13} \mathrm{C} \mathrm{NMR}\left(100 \mathrm{MHz}, \mathrm{CDCl}_{3}\right.$, only major isomer is shown, $\left.\delta\right): 19.2(C), 21.2$ (br, B-CH), $24.6\left(\mathrm{CH}_{3}\right), 24.7\left(\mathrm{CH}_{3}\right), 26.8\left(\mathrm{CH}_{3}\right), 33.9\left(\mathrm{CH}_{2}\right), 52.3\left(\mathrm{CH}_{3}\right), 62.8$ $\left(\mathrm{CH}_{2}\right), 83.2(\mathrm{C}), 111.0(\mathrm{CH}), 123.2(\mathrm{CH}), 127.5(\mathrm{CH}), 129.4(\mathrm{CH}), 134.0(\mathrm{C})$, $135.5(\mathrm{CH}), 153.9(\mathrm{C})$.

HRMS-ESI $(m / z):[\mathrm{M}+\mathrm{Na}]^{+}$calcd for $\mathrm{C}_{29} \mathrm{H}_{42} \mathrm{O}_{5} \mathrm{NBNaSi}, 545.28538$; found, 545.28624 .

Representative procedure for non-catalyzed allylation of aldehyde (Table 2, entry 1)

The allylboronate $(E)$-3a $(62.1 \mathrm{mg}, 0.2 \mathrm{mmol})$ was dissolved in toluene (0.4 mL). Then 4-nitrobenzaldehyde $(90.7 \mathrm{mg}, 0.6 \mathrm{mmol})$ was added to the mixture and stirred for $24 \mathrm{~h}$ at $50{ }^{\circ} \mathrm{C}$. The reaction mixture was quenched by a $\mathrm{CH}_{2} \mathrm{Cl}_{2}$ solution of triethanolamine $(10 \% \mathrm{v} / \mathrm{v}, 2.5 \mathrm{~mL})$. The crude product was purified by silica-gel chromatography (EtOAc:hexane $=10 / 90$ to $30 / 70$ ) to give the corresponding product (Z)-anti-4aa as a colorless oil, $82 \%$ isolated yield (55.2 $\mathrm{mg}, 0.164 \mathrm{mmol}$ ) and $E / Z=17: 83$. The $E-Z$ configuration of the products derided from the reaction of $(E)$ 3a and 4-nitrobenzaldehyde was confirmed from coupling constant of alkene moiety of the product. The $E / Z$ ratio of the anti-product was determined by ${ }^{1} \mathrm{H}$ NMR and HPLC analysis. The syn isomers of the products were not observed in ${ }^{1} \mathrm{H}$ NMR analysis of the crude reaction mixture.

${ }^{1} \mathrm{H}$ NMR (401 MHz, $\mathrm{CDCl}_{3}$, * indicates signals of the minor $E$-isomer, $\delta$ ): $0.82(\mathrm{t}, J=7.2 \mathrm{~Hz}, 3 \mathrm{H}), 0.83-0.89^{*}(\mathrm{~m}, 3 \mathrm{H}), 0.96-1.33(\mathrm{~m}, 6 \mathrm{H}), 1.65-1.81$ (m, 1H), 1.84-2.01 (m,1H), 3.19 (brs, 1H), 3.71* (s, 3H), $3.72(\mathrm{~s}, 3 \mathrm{H})$, 4.70 (brs, 1H), 4.86 (d, $J=7.6 \mathrm{~Hz}, 1 \mathrm{H}), 4.94^{*}(\mathrm{~d}, J=7.2 \mathrm{~Hz}, 1 \mathrm{H}), 5.00-5.12$ (m, 1H), 5.18-5.32 (m, 1H), $5.55(\mathrm{dt}, J=7.5,10.7 \mathrm{~Hz}, 1 \mathrm{H}), 7.54(\mathrm{~d}, J=8.4$ $\mathrm{Hz}, 2 \mathrm{H}), 8.20(\mathrm{~d}, J=8.8 \mathrm{~Hz}, 2 \mathrm{H})$.

${ }^{13} \mathrm{C}$ NMR (99 MHz, $\mathrm{CDCl}_{3}$, * indicates signals of the minor $E$-isomer, $\delta$ ): $13.8\left(\mathrm{CH}_{3}\right), 13.9^{*}\left(\mathrm{CH}_{3}\right), 22.3\left(\mathrm{CH}_{2}\right), 27.6\left(\mathrm{CH}_{2}\right), 28.5^{*}\left(\mathrm{CH}_{2}\right), 28.7\left(\mathrm{CH}_{2}\right)$, 31.1* $\left(\mathrm{CH}_{2}\right), 31.2\left(\mathrm{CH}_{2}\right), 32.1^{*}\left(\mathrm{CH}_{2}\right), 52.4\left(\mathrm{CH}_{3}\right), 54.2(\mathrm{CH}), 75.4(\mathrm{CH})$, $122.5(\mathrm{CH}), 123.1(\mathrm{CH}), 123.2^{*}(\mathrm{CH}), 127.2^{*}(\mathrm{CH}), 127.3(\mathrm{CH}), 135.9(\mathrm{CH})$, 136.0* $(\mathrm{CH}), 147.2(C), 148.1(C), 157.0(C)$

HRMS-ESI $(m / z):[M+N a]^{+}$calcd for $\mathrm{C}_{17} \mathrm{H}_{24} \mathrm{O}_{5} \mathrm{~N}_{2} \mathrm{Na}, 359.15771$; found, 359.15774

Allylation of 3-phenylpropionaldehyde with allylboronate (E)-3a (Table 2, entry 2)

The reaction was performed according to the representative procedure. The ${ }^{1} \mathrm{H}$ NMR and HPLC analysis gave $E / Z$ ratio of 23:77.

${ }^{1} \mathrm{H}$ NMR (401 MHz, $\mathrm{CDCl}_{3}$, * indicates signals of the minor $E$-isomer, $\delta$ ): $0.867(\mathrm{t}, J=7.0 \mathrm{~Hz}, 3 \mathrm{H}), 0.872^{*}(\mathrm{t}, J=6.8 \mathrm{~Hz}, 3 \mathrm{H}), 1.18-1.42(\mathrm{~m}, 6 \mathrm{H})$, $1.63-1.83(\mathrm{~m}, 2 \mathrm{H}), 1.98-2.23(\mathrm{~m}, 2 \mathrm{H}), 2.60-2.73(\mathrm{~m}, 1 \mathrm{H}), 2.78-2.91(\mathrm{~m}$, 1H), 3.67 (s, 3H), 3.71 (brs, 1H), 4.15* (brs, 1H), 4.45 (brs, 1H), 4.89 (brs, 1H), 5.04* (brs, $1 \mathrm{H}), 5.32$ (t, $J=10.0 \mathrm{~Hz}, 1 \mathrm{H}), 5.40^{*}$ (dd, $J=7.2,15.2 \mathrm{~Hz}$, $1 \mathrm{H}), 5.65(\mathrm{dt}, J=7.4,10.9 \mathrm{~Hz}, 1 \mathrm{H}), 5.70^{*}(\mathrm{dd}, J=6.4,10.9 \mathrm{~Hz}, 1 \mathrm{H}), 7.20(\mathrm{~d}$, $J=6.4 \mathrm{~Hz}, 2 \mathrm{H}), 7.24-7.32(\mathrm{~m}, 3 \mathrm{H})$.

${ }^{13} \mathrm{C}$ NMR (100 MHz, $\mathrm{CDCl}_{3}$, * indicates signals of the minor $E$-isomer, $\delta$ ): $13.9\left(\mathrm{CH}_{3}\right), 22.4^{*}\left(\mathrm{CH}_{2}\right), 22.5\left(\mathrm{CH}_{2}\right), 27.9\left(\mathrm{CH}_{2}\right), 28.7^{*}\left(\mathrm{CH}_{2}\right), 29.1\left(\mathrm{CH}_{2}\right)$, 31.3* $\left(\mathrm{CH}_{2}\right), 31.4\left(\mathrm{CH}_{2}\right), 32.1\left(\mathrm{CH}_{2}\right), 32.4^{*}\left(\mathrm{CH}_{2}\right), 35.0\left(\mathrm{CH}_{2}\right), 35.4^{*}\left(\mathrm{CH}_{2}\right)$, $52.2\left(\mathrm{CH}_{3}\right), 52.9(\mathrm{CH}), 73.4(\mathrm{CH}), 124.3(\mathrm{CH}), 124.6^{*}(\mathrm{CH}), 125.8(\mathrm{CH})$, $128.4(\mathrm{CH}), 135.2(\mathrm{CH}), 135.4(\mathrm{CH}), 141.7(\mathrm{C}), 156.7(\mathrm{C})$.

HRMS-ESI $(\mathrm{m} / \mathrm{z}):[\mathrm{M}+\mathrm{Na}]^{+}$calcd for $\mathrm{C}_{19} \mathrm{H}_{29} \mathrm{O}_{3} \mathrm{NNa}, 342.20404$; found, 342.20396 .

Allylation of cinnamaldehyde with allylboronate (E)-3a (Table 2, entry 3)

The reaction was performed according to the representative procedure. The ${ }^{1} \mathrm{H}$ NMR and HPLC analysis gave $E / Z$ ratio of $19: 81$.

${ }^{1} \mathrm{H}$ NMR (401 MHz, $\mathrm{CDCl}_{3}$, * indicates signals of the minor $E$-isomer, $\delta$ ): 0.78-0.92 (m, 3H), 1.18-1.42 (m, 6H), 2.00-2.22 (m, 2H), 2.55 (brs, 1H), 3.69 (s, 3H), 4.42 (brs, 1H), 4.63 (brs, 1H), 4.94 (brs, 1H), 5.35 (t, J = 10.2 $\mathrm{Hz}, 1 \mathrm{H}), 5.44^{*}(\mathrm{dd}, J=6.4,15.2 \mathrm{~Hz}, 1 \mathrm{H}), 5.66(\mathrm{dt}, J=7.5,11.1 \mathrm{~Hz}, 1 \mathrm{H}), 6.17$ $(\mathrm{dd}, J=6.8,16.0 \mathrm{~Hz}, 1 \mathrm{H}), 6.66(\mathrm{~d}, J=16.0 \mathrm{~Hz}, 1 \mathrm{H}), 7.20(\mathrm{~m}, 5 \mathrm{H})$.

${ }^{13} \mathrm{C} \mathrm{NMR} \mathrm{(100} \mathrm{MHz}, \mathrm{CDCl}_{3}$, * indicates signals of the minor $E$-isomer, $\delta$ ): $13.92\left(\mathrm{CH}_{3}\right), 13.95^{*}\left(\mathrm{CH}_{3}\right), 22.4\left(\mathrm{CH}_{2}\right), 27.9\left(\mathrm{CH}_{2}\right), 28.7^{*}\left(\mathrm{CH}_{2}\right), 29.1\left(\mathrm{CH}_{2}\right)$, 31.3* $\left(\mathrm{CH}_{2}\right), 31.4\left(\mathrm{CH}_{2}\right), 32.3^{*}\left(\mathrm{CH}_{2}\right), 52.25\left(\mathrm{CH}_{3}\right), 53.33(\mathrm{CH}), 74.7^{*}(\mathrm{CH})$, 
$75.0(\mathrm{CH}), 124.4(\mathrm{CH}), 124.9^{*}(\mathrm{CH}), 126.5(\mathrm{CH}), 127.7(\mathrm{CH}), 127.8(\mathrm{CH})$ 127.9* $(\mathrm{CH}), 128.5(\mathrm{CH}), 131.8^{*}(\mathrm{CH}), 132.1(\mathrm{CH}), 134.8^{*}(\mathrm{CH}), 135.2$ $(\mathrm{CH}), 136.4(\mathrm{C}), 156.9(\mathrm{C})$.

HRMS-ESI $(\mathrm{m} / \mathrm{z})$ : $[\mathrm{M}+\mathrm{Na}]^{+}$calcd for $\mathrm{C}_{19} \mathrm{H}_{27} \mathrm{O}_{3} \mathrm{NNa}, 340.18823$; found, 340.18831 .

\section{Allylation of 4-nitrobenzaldehyde with allylboronate $(E)-3 c$ (Table 2, entry 4)}

The reaction was performed according to the representative procedure. The ${ }^{1} \mathrm{H}$ NMR and HPLC analysis gave $E / Z$ ratio of $17: 83$

${ }^{1} \mathrm{H}$ NMR (401 MHz, $\mathrm{CDCl}_{3}, *$ indicates signals of the minor $E$-isomer, $\delta$ ): $0.82(\mathrm{t}, J=7.2 \mathrm{~Hz}, 3 \mathrm{H}), 0.85^{*}(\mathrm{t}, J=6.8 \mathrm{~Hz}, 3 \mathrm{H}), 0.96-1.32(\mathrm{~m}, 6 \mathrm{H}), 1.67-$ $1.83(\mathrm{~m}, 1 \mathrm{H}), 1.84-2.01(\mathrm{~m}, 1 \mathrm{H}), 3.20(\mathrm{brs}, 1 \mathrm{H}), 4.73(\mathrm{brs}, 1 \mathrm{H}), 4.91$ (d, J= $6.8 \mathrm{~Hz}, 1 \mathrm{H}), 4.96-5.08(\mathrm{~m}, 1 \mathrm{H}), 5.08-5.19(\mathrm{~m}, 2 \mathrm{H}), 5.23(\mathrm{t}, J=10.2 \mathrm{~Hz}$ $1 \mathrm{H}), 5.26-5.31^{*}(\mathrm{~m}, 1 \mathrm{H}), 5.56(\mathrm{dt}, J=7.4,10.8 \mathrm{~Hz}, 1 \mathrm{H}), 7.54(\mathrm{~m}, 5 \mathrm{H}), 7.50$ $(\mathrm{d}, J=8.0 \mathrm{~Hz}, 2 \mathrm{H}), 8.16(\mathrm{~d}, J=8.8 \mathrm{~Hz}, 2 \mathrm{H}), 8.17^{*}(\mathrm{~d}, J=8.4 \mathrm{~Hz}, 2 \mathrm{H})$.

${ }^{13} \mathrm{C}$ NMR (99 $\mathrm{MHz}, \mathrm{CDCl}_{3}$, * indicates signals of the minor $E$-isomer, $\delta$ ): $13.86\left(\mathrm{CH}_{3}\right), 13.92 *\left(\mathrm{CH}_{3}\right), 22.4\left(\mathrm{CH}_{2}\right), 27.7\left(\mathrm{CH}_{2}\right), 28.4^{*}\left(\mathrm{CH}_{2}\right), 28.7\left(\mathrm{CH}_{2}\right)$, 31.1* $\left(\mathrm{CH}_{2}\right), 31.2\left(\mathrm{CH}_{2}\right), 32.2 *\left(\mathrm{CH}_{2}\right), 54.3(\mathrm{CH}), 67.2\left(\mathrm{CH}_{2}\right), 75.6(\mathrm{CH})$, $122.5(\mathrm{CH}), 123.1(\mathrm{CH}), 127.2^{*}(\mathrm{CH}), 127.3(\mathrm{CH}), 128.1(\mathrm{CH}), 128.3(\mathrm{CH})$, $128.5(\mathrm{CH}), 136.0(\mathrm{CH}), 136.1^{*}(\mathrm{CH}), 147.2(\mathrm{C}), 147.9(\mathrm{C}), 156.4(\mathrm{C})$.

HRMS-ESI $(m / z):[\mathrm{M}+\mathrm{Na}]^{+}$calcd for $\mathrm{C}_{23} \mathrm{H}_{28} \mathrm{O}_{5} \mathrm{~N}_{2} \mathrm{Na}, 435.18894$; found, 435.18904.

\section{Allylation of 4-nitrobenzaldehyde with allylboronate $(E)-3$ j (Table 2 ,} entry 5)

The reaction was performed according to the representative procedure. The ${ }^{1} \mathrm{H}$ NMR and HPLC analysis gave $E / Z$ ratio of $27: 73$.

${ }^{1} \mathrm{H}$ NMR (399 MHz, $\mathrm{CDCl}_{3}$, * indicates signals of the minor $E$-isomer, $\delta$ ): 2.15-2.35 (m, 2H), 3.28-3.46 (m, 2H), 3.55 (brs, 1H), 3.65 (s, 3H), 3.71* $(\mathrm{s}, 3 \mathrm{H}), 4.46(\mathrm{~s}, 2 \mathrm{H}), 4.47^{*}(\mathrm{~s}, 2 \mathrm{H}), 4.66(\mathrm{brs}, 1 \mathrm{H}), 4.97$ (brs, 2H), 5.32* (dd, $J=7.0,15.4 \mathrm{~Hz}, 1 \mathrm{H}), 5.39(\mathrm{dd}, J=9.4,10.6 \mathrm{~Hz}, 1 \mathrm{H}), 5.58^{*}(\mathrm{q}, J=7.4 \mathrm{~Hz}$, $1 \mathrm{H}), 5.65(\mathrm{dt}, J=7.8,10.9 \mathrm{~Hz}, 1 \mathrm{H}), 7.24-7.40(\mathrm{~m}, 5 \mathrm{H}), 7.44^{*}(\mathrm{~d}, J=8.8 \mathrm{~Hz}$, $2 \mathrm{H}), 7.49(\mathrm{~d}, J=8.8 \mathrm{~Hz}, 2 \mathrm{H}), 8.11-8.18(\mathrm{~m}, 2 \mathrm{H})$.

${ }^{13} \mathrm{C}$ NMR (99 $\mathrm{MHz}, \mathrm{CDCl}_{3}$, * indicates signals of the minor $E$-isomer, $\delta$ ): $28.5\left(\mathrm{CH}_{2}\right), 32.7^{*}\left(\mathrm{CH}_{2}\right), 52.5(\mathrm{CH}), 54.5(\mathrm{CH}), 58.7^{*}(\mathrm{CH}), 68.7\left(\mathrm{CH}_{2}\right)$ 69.0* $\left(\mathrm{CH}_{2}\right), 72.96^{*}\left(\mathrm{CH}_{2}\right), 73.02\left(\mathrm{CH}_{2}\right), 75.3\left(\mathrm{CH}_{3}\right), 123.2(\mathrm{CH}), 123.3 *$ $(\mathrm{CH}), 125.5^{*}(\mathrm{CH}), 125.6(\mathrm{CH}), 127.2^{*}(\mathrm{CH}), 127.4(\mathrm{CH}), 127.7(\mathrm{CH}), 128.4$ $(\mathrm{CH}), 132.0^{*}(\mathrm{CH}), 132.1(\mathrm{CH}), 137.8(\mathrm{C}), 138.0^{*}(\mathrm{C}), 147.3(\mathrm{C}), 147.8^{*}(\mathrm{C})$, $148.1(C), 157.0(C)$.

HRMS-ESI $(m / z)$ : $[\mathrm{M}+\mathrm{Na}]^{+}$calcd for $\mathrm{C}_{21} \mathrm{H}_{24} \mathrm{O}_{6} \mathrm{~N}_{2} \mathrm{Na}$, 423.15256; found, 423.15266 .

\section{Acknowledgments}

This study was financially supported by the MEXT (Japan) program (Strategic Molecular and Materials Chemistry through Innovative Coupling Reactions) of Hokkaido University, as well as the JSPS (KAKENHI Grant Numbers 15H03804 and 15K13633).

\section{Supporting Information}

YES

\section{Primary Data}

NO

\section{References and Notes}

(1) (a) Lee, H. -S.; Kang, S. H. Synlett 2004, 1673-1685. (b) Bergmeier S. C. Tetrahedron 2000, 56, 2561-2576. (c) Karjalainen, O. K.; Koskinen, A. M. P. Org. Biomol. Chem. 2012, 10, 4311-4326.

(2) (a) Tao, B.; Schlingloff, G.; Sharpless, K. B. Tetrahedron Lett. 1998, 39, 2507-2510. (b) Demko, Z. P.; Bartsch, M.; Sharpless, K. B. Org. Lett. 2000, 2, 2221-2223. (c) Wang, C.; Yamamoto, H. J. Am. Chem. Soc. 2014, 136, 6888-6891.

(3) (a) Lombardo, M.; Mosconi, E.; Pasi, F.; Petrini, M. A.; Trombini, C. J. Org. Chem. 2007, 72, 1834-1837. (b) Keinicke, L.; Fristrup, P.; Norrby, P. A.; Madsen, R. J. Am. Chem. Soc. 2005, 127, 1575615761. (c) List, B.; Pojarliev, P.; Biller, W. T.; Martin, H. J. J. Am. Chem. Soc. 2002, 124, 827-833. (d) Matsunaga, S.; Yoshida, T.; Morimoto, H.; Kumagai, N.; Shibasaki, M. J. Am. Chem. Soc. 2004, 126, 8777-8785. (e) Enders, D.; Grondal, C.; Vrettou, M.; Raabe, G. Angew. Chem. Int. Ed. 2005, 44, 4079-4083. (f) Trost, B. M.; Jaratjaroonphong, J.; Reutrakul, V. J. Am. Chem. Soc. 2006, 128, 2778-2779. (g) Carswell, E. L.; Snapper, M. L.; Hoveyda, A. H. Angew. Chem. Int. Ed. 2006, 45, 7230-7233. (h) Ramasastry, S. S. V.; Zhang, H.; Tanaka, F.; Barbas, C. F. III. J. Am. Chem. Soc. 2006, 129, 288-289. (i) Zhong, Y.-W.; Dong, Y.-Z.; Fang, K.; Izumi, K.; Xu, M.-H.; Lin, G.-Q. J. Am. Chem. Soc. 2005, 127, 11956-11957. (j) Skucas, E.; Zbieg, J. R.; Krische, M. J. J. Am. Chem. Soc. 2009, 131, 5054-5055.

(4) (a) Barrett, A. G. M.; Seefeld, M. A. Tetrahedron 1993, 49, 7857. (b) Barrett, A. G. M.; Seefeld, M. A. J. Chem. Soc. Chem. Commun. 1993, 339. (c) Barrett, A. G. M.; Seefeld, M. A.; Williams, D. J. J. Chem. Soc. Chem. Commun. 1994, 1053. (d) Barrett, A. G. M.; Seefeld, M. A.; White, A. J. P.; Williams, D. J. J. Org. Chem. 1996, 61, 2677.

(5) (a) Lessard, S.; Peng, F.; Hall, D. G. J. Am. Chem. Soc. 2009, 131, 9612-9613. (b) Ding, J.; Hall, D. G. Angew. Chem. Int. Ed. 2013, 52, $1-6$.

(6) Yamamoto, E.; Takenouchi, Y.; Ozaki, T. Miya, T.; Ito, H. J. Am. Chem. Soc. 2014, 136, 16515-16521.

(7) (a) Ito, H.; Kawakami, C.; Sawamura, M. J. Am. Chem. Soc. 2005, 127, 16034-16035. (b) Ito, H.; Sasaki, Y.; Sawamura, M. J. Am. Chem. Soc. 2008, 130, 15774-15775. (c) Ito, H.; Okura, T.; Matsuura, K.; Sawamura, M. Angew. Chem. Int. Ed. 2010, 49, 560563. (d) Guzman-Martinez, A.; Hoveyda, A. H. J. Am. Chem. Soc. 2010, 132, 10634-10637.

(8) (a) Ito, H.; Kunii, S.; Sawamura, M. Nat. Chem. 2010, 2, 972-976. (b) Park, J. K.; Lackey, H. H.; Ondrusek, B. A.; McQuade, D. T. J. Am. Chem. Soc. 2011, 133, 2410-2413. (c) Park, J. K.; McQuade, D. T. Angew. Chem. Int. Ed. 2012, 51, 2717-2721.

(9) Ito, H.; Toyoda, T.; Sawamura, M. J. Am. Chem. Soc. 2010, 132, 5990-5992.

(10) Zhong, C.; Kunii, S.; Kosaka, Y.; Sawamura, M.; Ito, H. J. Am. Chem. Soc. 2010, 132, 11440-11442.

(11) Zhao, J.; Szabó, K. J. Angew. Chem. Int. Ed. 2015, 54, 1-6.

(12) Lee, H.; Han, H. T.; Yun, J. ACS Catal. 2016, 6, 6487-6490.

(13) (a) Kano, T.; Yurino, T.; Asakawa, D.; Maruoka, K. Angew. Chem. Int. Ed. 2013, 125, 5642-5644. (b) Kano, T.; Kobayashi, R.; Maruoka, K. Angew. Chem. Int. Ed. 2015, 54, 1-5. 